Trans

continentales
Transcontinentales

Sociétés, idéologies, système mondial

5 | 2007

Le défi sanitaire

\title{
Pratiques sociales, politiques de santé publique et nouvelles épidémies en Egypte
}

À propos de l'hépatite $C$ et de la grippe aviaire

Social Behavior, Public Health Policies and New Epidemics in Egypt: The Case of

Hepatitis $C$ and Avian Influenza

Saadia Radi

\section{OpenEdition}

\section{Journals}

Édition électronique

URL : http://journals.openedition.org/transcontinentales/701

DOI : 10.4000/transcontinentales.701

ISBN : 978-2-7351-1563-1

ISSN : 1775-397X

Éditeur

Editions de la maison des sciences de l'homme

Édition imprimée

Date de publication : 31 décembre 2007

Pagination : 57-69

ISBN : 978-2-200-92397-6

ISSN : 1950-1684

Référence électronique

Saadia Radi, «Pratiques sociales, politiques de santé publique et nouvelles épidémies en Egypte », Transcontinentales [En ligne], 5 | 2007, document 4, mis en ligne le 15 avril 2011, consulté le 07 septembre 2020. URL : http://journals.openedition.org/transcontinentales/701 ; DOI : https://doi.org/ 10.4000/transcontinentales.701 


\title{
Pratiques sociales, politiques de santé publique et nouvelles épidémies en Égypte
}

\author{
À propos l'hépatite $\mathrm{C}$ et de la grippe aviaire
}

\section{Saadia Radi}

L'une des caractéristiques du monde actuel est paradoxalement d'être marqué par le risque ${ }^{1}$, c'est-à-dire par une forme de «non savoir» créé par la science. La science concède qu'il y a beaucoup de choses qu'elle ne sait pas. Ce "non savoir» a des conséquences sur les décisions politiques, puisque les experts placent les décideurs des politiques publiques devant des incertitudes qui augmentent la perception du risque. La crise de la vache folle en Grande-Bretagne en est une illustration connue ${ }^{2}$. Cette liaison négative entre décideurs politiques et experts s'accompagne généralement d'une liaison revendicative entre population et décideurs politiques, la population considérant que ceux-ci doivent la préserver des risques. C'est l'une des leçons politiques de l'épidémie du sida ${ }^{3}$. Toutefois, les systèmes de santé européens possèdent suffisamment de ressources pour chercher à mettre en place des réponses sophistiquées, à la fois politiques, sociales et scientifiques, comme en témoigne la formulation du principe de précaution ${ }^{4}$.

Il n'en est pas de même dans les pays du Sud, où les nombreuses carences institutionnelles ne permettent pas la mise en place de telles réponses ${ }^{5}$. Le risque y est toujours présent, mais son traitement s'avère bien plus problématique. De plus, il ne résulte pas seulement d'un "non savoir" mais également d'une absence de

1 - Voir U. Beck, La société du risque. Sur la voie d'une autre modernité, Paris, Aubier, 2001.

2 - Voir E. Seguin : «La crise de la vache folle au Royaume-Uni. Quelques explications », Revue française de science politique, vol.52, $\mathrm{n}^{\circ}$ 2-3, 2002 et «L'évaluation britannique du risque de transmission de la maladie de la vache folle aux humains ", Revue française d'administration publique, $\mathrm{n}^{\circ}$ 103, 2002/2003.

3 - Voir N. Dodier, Les leçons politiques de l'épidémie de sida, Paris, Éditions de l'EHESS, 2003.

4 - Voir O. Godard, «Le principe de précaution comme norme de l'action publique, ou la proportionnalité en question", Revue économique, vol. 54, nº 6, 2003.

5 - Voir A. Hamdouch et M.-H. Depret, «Carences institutionnelles et rationnement de l'accès à la santé dans les pays en développement : repères et enjeux», Mondes en développement, vol. 33, n 131, 2005. 
moyens, tant dans le domaine proprement médical du traitement, que dans ceux des politiques de prévention et de la protection sociale offrant des possibilités d'accès aux soins. À l'instar des sociétés du Nord, ces trois aspects sont mêlés, mais en revanche au Sud, la population n'adopte pas toujours une attitude critique collective - revendicative - envers les pouvoirs publics ${ }^{6}$. En outre, contrairement à la situation prévalant au Nord, les politiques publiques de santé sont, au Sud, très dépendantes de l'aide internationale et donc de l'évolution des politiques de développement ${ }^{7}$. Vues du Sud, ces politiques peuvent apparaître inégalitaires et insuffisantes. Elles constituent aussi un enjeu non négligeable pour le Nord, puisque des maladies émergentes peuvent se développer au Sud, y connaître des mutations et se répandre au Nord. La question des politiques de santé publique du Sud face aux nouvelles épidémies apparaît ainsi particulièrement importante, d'un point de vue local comme d'un point de vue global.

Une épidémie n'est pas uniquement un phénomène biologique - même si, au départ, il s'agit bien d'une question virale -, puisque la qualité même de la réponse médicale dépend d'une série complexe de facteurs sociaux. En même temps, on ne soutiendra pas qu'elle est soluble dans le social, ni qu'importent seuls les dispositifs symboliques. C'est un mal réel qui est en jeu, de sorte que le social varie aussi en fonction de la réponse biomédicale, comme le montre le tournant de la trithérapie dans la cure du sida ${ }^{8}$. Le social serait plutôt le contexte écologique de l'épidémie et les politiques publiques des éléments de structuration de ce contexte. Étudier les politiques publiques ne nous éloigne donc pas du problème proprement virologique. Bien sûr, ces politiques nous apprennent des choses sur la société, mais elles nous les apprennent en relation avec la maladie dont le monde humain est la niche écologique.

Dans les limites que nous venons de décrire, on peut dire qu'une maladie ou une épidémie ont une "carrière sociale» et c'est, en fait, parce que cette carrière sociale est aussi une carrière "écologique» que les sciences sociales participent à la constitution du savoir et de l'expertise sur la santé. Deux dispositifs paraissent déterminants : a) les conditions d'émergence et de réception d'une atteinte et $b$ ) la structure et l'état du système de santé et de protection sociale. Par réception de l'atteinte, on entend la façon dont on prend connaissance de l'existence de l'épidémie et de ses caractéristiques - car ni l'une ni l'autre chose ne sont un savoir immédiat - et l'effet que cette connaissance produit sur les décideurs et les populations. Cet effet sera mis en relation avec l'état et la structure du système de santé, dans la mesure où il traduit aussi un jugement sur ses capacités et où il peut constituer une impulsion pour le modifier. On peut ne pas avoir peur d'une épidémie, parce que le système

6 - Voir S. Radi : «Débats de presse, scandale et mise en place d'une politique de prévention : à propos de l'hépatite C en Égypte», Revue d'épidémiologie et de santé publique, vol. 54, 2006 et "L'hépatite $\mathrm{C}$ et les défaillances du système égyptien de santé publique. Itinéraires thérapeutiques et solutions palliatives», Égypte-Monde arabe, n 4, 2007.

7 - S. Tizio, «État de santé et système de soins dans les pays en développement : la contribution des politiques de santé au développement durable», Mondes en développement, vol.32, n²127, 2004.

8 - C. Herzlich, "Vingt ans après... l'évolution d'une épidémie», Études, t. 396, n² 2, 2002, p. 192 et suiv. 
de santé paraît à même d'y pallier. On peut, au contraire, avoir peur, parce que l'on s'aperçoit qu'il n'en est pas capable. Ces deux situations peuvent entraîner des attitudes différentes de la part de la population et de la part des pouvoirs publics. Ces attitudes, comme le type de relation entre les pouvoirs publics et la population, auront des conséquences sur la carrière de l'épidémie.

L'Égypte connaît, depuis les années 1990, une épidémie d'hépatite C frappant plus de $10 \%$ de la population et semble aujourd'hui en première ligne de l'épidémie de grippe aviaire. Elle offre donc un cas de figure particulièrement intéressant pour revenir sur l'apport des sciences sociales à l'analyse des questions de santé et sur la relation entre les politiques publiques et la "carrière» des maladies. On évoquera d'abord quelques traits généraux de la question. On s'intéressera ensuite aux conditions sociales de l'émergence des épidémies d'hépatite $\mathrm{C}$ et de grippe aviaire en Égypte ainsi qu'aux premières réponses fournies par le système local de santé. On conclura en soulignant l'importance de l'organisation matérielle de ce système et la nécessité heuristique de l'étudier davantage.

\section{Une approche fonctionnelle : les pratiques sociales et leurs contextes}

Les sciences sociales s'intéressant aux questions de santé ont, de manière bien compréhensible, eu tendance à prendre du champ par rapport à la question médicale et biologique stricto sensu, afin de préserver leur autonomie. C'est la position de Marc Augé 9 . On la retrouve aussi chez des médecins venus à l'anthropologie médicale qui veulent assurer l'autonomie de leur approche en la fondant dans un ordre de préoccupation extérieur à la biologie, comme Didier Fassin, qui considère la santé comme un espace politique ${ }^{10}$. Toutefois, l'avantage de l'approche de Fassin est de se centrer sur le fonctionnement du système de santé que le détour par le politique lui permet de mieux analyser. Un deuxième avantage de cette approche réside dans le fait qu'en mettant la question du pouvoir au centre des études sur la santé, elle permet au chercheur d'échapper au découpage opposant les "sociétés traditionnelles» et les "sociétés moderne», les pratiques du passé et celles du présent.

En effet, bien que la représentation de la maladie dans tel ou tel contexte culturel soit toujours un domaine pertinent d'analyse, elle ne peut suffire. Il faut aussi interroger les politiques publiques de la santé, ses lieux et ses hommes, et les relations que les malades entretiennent avec elles, parce que cet aspect des choses fait partie de son monde quotidien ${ }^{11}$. Si des malades n'ont pas recours au système biomédical institutionnel, ce n'est pas simplement parce qu'ils auraient des raisons «culturelles » de l'éviter. Il faut s'interroger sur ce que le système de santé public offre aux citoyens, sur les politiques de santé publique et sur la manière dont les malades sont traités dans les institutions qui en relèvent, ainsi que sur les secours extérieurs qui peuvent

9 - Voir M. Augé, "L'anthropologie de la maladie», L'Homme, vol. 26, n 1-2, 1986.

10 - D. Fassin, L'espace politique de la santé. Essai de généalogie, Paris, PUF, 1996.

11 - Voir S. Fainzang, Pour une anthropologie de la maladie en France : un regard africaniste, Paris, Éditions de l'EHESS, 1989 
s'offrir. En outre, les différences en matière de santé et de maladie ne relèvent pas seulement de la culture locale ou des politiques globales de santé, mais aussi de la position que chaque groupe occupe dans sa propre société, comme le note Bernard Hours : "Le développement ne se pose plus comme un problème entre les nations mais entre les groupes sociaux dans le "village planétaire" ${ }^{12}$ ».

Si un riche Égyptien peut se soigner exactement comme un Américain (a fortiori s'il peut se faire traiter aux États-Unis), le gouvernement égyptien ne peut appliquer à l'Égypte les mesures de santé publique mises en place dans les pays du Nord. Les groupes sociaux les moins favorisés sont donc, eux, totalement dépendants des conditions locales. Cette double asymétrie appelle en conséquence à questionner les modes de fonctionnement qu'impliquent les différences de statut entre les groupes sociaux et entre les sociétés. Elle permet aussi de s'intéresser aux conduites des gens vis-à-vis de la maladie et des systèmes de soins, sans perdre de vue leur insertion dans des systèmes plus larges.

\section{L'émergence des épidémies}

Si l'émergence d'une épidémie est fondamentalement biologique, sa prise en considération passe par des schèmes d'interprétation complexes, car au départ l'épidémie n'est pas nécessairement identifiée comme telle, et ses conséquences à moyen terme ne sont pas précisément évaluées. Cette incertitude est une des caractéristiques majeures de notre époque ${ }^{13}$, qui succède à un temps où l'on pensait pouvoir cerner et vaincre le mal ${ }^{14}$. Une perception mise à mal depuis l'épidémie du sida, dont l'histoire biologique, médicale et sociale ${ }^{15}$ a fortement contribué à susciter le doute face aux nouvelles épidémies. Les craintes qui en résultent sont en effet alimentées par l'apparition de maladies émergentes, ces "virus qui détruisent les hommes ${ }^{16}$ ", et par les mutations de maladies animales en maladies humaines ${ }^{17}$, dont la carrière biologique et publique dessine une nouvelle carte virale ${ }^{18}$.

Les pays en développement partagent les mêmes craintes que les pays développés en ce domaine, parce qu'ils sont tributaires de la même médecine et de la même science, sans bénéficier des mêmes moyens et des mêmes ressources. Les crises épidémiologiques n'y prennent donc pas exactement la même forme et n'y ont pas les mêmes conséquences. En Grande-Bretagne, la maladie de la «vache folle» a pu entraîner l'abattage d'un nombre considérable de bovins. Ces abattages,

12 - B. Hours, "Pour une anthropologie de la santé en sociétés», in B. Hours (dir.), Systèmes et politiques de santé. De la santé publique à l'anthropologie, Paris, Karthala, 2001, p. 15.

13 - Voir U. Beck, op. cit., 2001.

14 - Voir J.-P. Bado, Médecine coloniale et grandes endémies en Afrique, Paris, Karthala, 1996; F. Delaporte, Histoire de la fièvre jaune. Naissance de la médecine tropicale, Paris, Payot, 1989.

15 - Voir S. Epstein, Histoire du sida, Paris, Les Empêcheurs de penser en rond, 2001 et M. Grmek, Histoire du sida, Paris, Payot, 1989.

16 - C. Chastel, Ces virus qui détruisent les hommes, Paris, Ramsey, 1996.

17 - J.-P. Cassuto, De la maladie de la vache folle à celle de Creutzfeldt-Jakob, Paris, Odile Jacob, 1999.

18 - N. Gualde, Épidémies, la nouvelle carte, Paris, Desclée de Brouwer, 2002. 
qui répondaient à des motifs de santé publique et à des raisons politiques ${ }^{19}$, s'accompagnaient d'indemnisations. En Égypte, une politique d'abattage des volailles domestiques pour prévenir la grippe aviaire ne s'accompagne pas d'indemnisation et met en cause la survie des ménages, du moins est-ce la perception des petits éleveurs.

Si l'on a pu montrer que le sida en France était à la fois porteur de leçons politiques ${ }^{20}$ et créateur de mouvements sociaux ${ }^{21}$, il n'en est pas de même en Égypte avec l'hépatite $\mathrm{C}$, malgré une forte couverture de presse ${ }^{22}$, probablement parce que la nature autoritaire du régime bloque la protestation sociale et parce que la pauvreté des ressources conduit les malades à attendre peu de choses de l'État. Bien sûr, les États autoritaires dans les pays en développement ont aussi intérêt à satisfaire une part de la demande sociale en contrepartie de l'absence de démocratie ${ }^{23}$, mais à l'inverse, leur manière de gérer une crise opère dans un contexte où les pressions des citoyens sont faibles, ou de peu d'impact.

Si le niveau des connaissances et l'accès aux informations médicales sont variables à travers le monde, on peut considérer que, dans le cas des maladies émergentes, la faiblesse des informations est un mal partagé entre pays développés et pays en développement. Face à un même inconnu, c'est d'abord la "gestion de crise» qui semble en jeu : comment réagit-on et quels moyens peut-on mobiliser pour le faire? Ici aussi jouent la question des ressources, celle des moyens effectivement mis en œuvre et celle de la pression sociale face à la crise. La crise française de la canicule a pu laisser le sentiment que la réaction des pouvoirs publics était insuffisante et que l'appareil sanitaire était inadapté ${ }^{24}$. Cette insuffisance et cette inadaptation sont des faits, mais aussi des jugements par rapport à des attentes de protection maximale. Les attentes dans les pays en développement sont d'une nature différente: on y espère la "bonne santé» comme partout ailleurs, mais on n'escompte pas de l'État une performance comparable. Un bon résultat, dans un tel contexte, peut être simplement défini par l'accès à un médecin et à des médicaments.

L'émergence des épidémies ravive le sentiment du risque, dans une forme localement déterminée moins par les paramètres strictement culturels que par les informations disponibles et par les attentes de la population en matière de santé publique. Le plus souvent, la population espère ce qu'elle pense pouvoir

19 - Voir E. Seguin, art. cit., 2002 et art. cit. 2002/2003.

20 - Voir P. Pinell, Une épidémie politique. La lutte contre le sida en France (1981-1996), Paris, PUF, 2002 et N. Dodier, op. cit., 2003.

21 - Voir F. Buton, "Sida et politique : saisir les formes de la lutte», Revue française de science politique, vol. 55, $\mathrm{n}^{\circ}$ 5-6, 2005.

22 - S. Radi, «Débats de presse, scandale et mise en place d'une politique de prévention : à propos de l'hépatite C en Égypte», Revue d'épidémiologie et de santé publique, vol. 54, 2006.

23 - G. Hermet, «Un régime à pluralisme limité. À propos de la gouvernance démocratique», Revue française de science politique, vol. 54, $\mathrm{n}^{\circ} 1,2004$.

24 - Voir M. Bungener, "Canicule estivale : la triple vulnérabilité des personnes âgées", Mouvements, $\mathrm{n}^{\circ} 32,2004$ et M. Milet, "Cadre de perception et luttes d'imputation dans la gestion de crise l'exemple de la canicule d'août $2003 »$, Revue française de science politique, vol. 55, n 4, 2005. 
raisonnablement espérer et proportionne ses attentes à la nature de cette espérance. En ce sens, le sentiment du risque et la peur ne sont pas des absolus; ils sont créés par la perception de la situation. «Perception de la situation» n'équivaut pas à « déterminisme culturel ».

\section{Les politiques publiques}

et le système de santé et de protection sociale

Les pays en développement ne sont pas entrés dans ce que Raymond Massé nomme la «nouvelle santé publique», qui intègre une forte dimension citoyenne ${ }^{25}$. Dans ces pays, la dimension citoyenne est pour l'essentiel absente des politiques de santé publique et, plus largement, des systèmes de santé et de protection sociale. La "cité civique», comme l'appelle Stéphane Tizio, est atone ${ }^{26}$. Il en découle plusieurs conséquences, holistes et individuelles.

Du point de vue holiste, la faiblesse du citoyen a comme conséquence une plus grande autonomie des décideurs politiques. Elle augmente aussi leur incertitude : si le système de soins ne fonctionne pas suffisamment bien, mais si une remise en cause de son fonctionnement peut entraîner le mécontentement de groupes favorables aux décideurs (ou simplement provoquer des problèmes difficiles à résoudre), il peut être tentant pour ceux-ci de s'abstenir. Le laps de temps qui s'est écoulé en Égypte entre la connaissance d'une épidémie d'hépatite - d'abord définie comme "ni A ni $\mathrm{B}$ », puis appelée " $\mathrm{C}$ » - et la mise en place de politiques publiques inadéquates s'explique par le fait que les conséquences mal évaluées de l'épidémie paraissaient moins gênantes qu'une remise en cause de la politique de santé à la mesure de l'importance de l'épidémie. Admettre l'existence d'une épidémie, c'est en effet, pour les décideurs politiques, se placer dans la situation de mettre en place des recours. Les autorités égyptiennes ne le firent que sous la contrainte extérieure, lorsque les pays du Golfe exigèrent de tout immigré égyptien la production d'un certificat médical constatant l'absence d'hépatite $\mathrm{C}^{27}$.

Du point de vue individuel, la faiblesse du citoyen entraîne une individualisation de la maladie comme le souligne $\operatorname{Tizio}^{28}$. Cette individualisation aboutit à la mise en place d'itinéraires thérapeutiques variés, impliquant une fuite hors du système de santé et le recours à des soins palliatifs ${ }^{29}$. Si les soins relevant du système biomédical ne sont pas ouverts aux malades, ils recourent à d'autres soins : médecines traditionnelles, médecines par les plantes, médecines «religieuses», etc. Certes, cette fuite allège dans un premier temps le système de santé public, mais il est

25 - R. Massé, «La santé publique comme projet politique et projet individuel», in B. Hours (dir.), Systèmes et politiques de santé. De la santé publique à l'anthropologie, Paris, Karthala, 2001.

26 - S. Tizio, «Trajectoires socioéconomiques de la régulation des systèmes de santé dans les pays en développement : une problématique institutionnelle», Mondes en développement, vol. 33, n 131, 2005.

27 - Voir S. Radi, art. cit., 2006.

28 - S. Tizio, art. cit., 2005.

29 - On entend ici par «soins palliatifs» des soins qui, sans guérir le malade, rendent la maladie plus supportable - sans référence à une phase de fin de vie. Sur ces itinéraires thérapeutiques, voir S. Radi, art. cit., 2007. 
facile de deviner les conséquences néfastes qui en découlent, non seulement en matière de soins, mais aussi en ce que cette esquive n'incite pas, paradoxalement, à réformer le système de santé. L'individualisation peut aussi évidemment, favoriser les épidémies parce qu'elle implique un déficit d'information et donc une sousestimation des conduites risquées.

\section{L'émergence de I'hépatite $\mathrm{C}$ et de la grippe aviaire}

L'hépatite $\mathrm{C}$ est une maladie virale du foie; les porteurs du virus risquent de développer une affection hépatique chronique, une cirrhose et un cancer du foie. Le virus de l'hépatite C (VHC) a été isolé en 1989. Avant cette date, on parlait $\mathrm{d}^{\prime}$ «hépatite non A non B». L'Organisation mondiale de la santé (OMS) estime que 170 millions de personnes seraient atteintes, soit $3 \%$ de la population mondiale, et que chaque année 3 à 4 millions de personnes seraient infectées ${ }^{30}$. Cette maladie existe partout dans le monde, sa prévalence varie selon les pays et selon les régions : elle est de 5,3\% en Afrique, de 1,7\% en Amérique, de 4,6\% en Méditerranée orientale, de 1, $03 \%$ en Europe, de 2,15 \% en Asie du Sud-est, et de 3,9\%, dans le Pacifique occidental. Le VHC se transmet principalement par le contact direct du sang contaminé, lors d'une transfusion sanguine, d'une dialyse ou par la réutilisation de seringues ou de matériel médical mal stérilisé. La particularité de cette maladie est d'être asymptomatique. De nombreuses personnes atteintes par le virus l'ignorent, et la plupart de celles qui savent qu'elles sont malades l'ont découvert par hasard ou lorsque leur état de santé s'est aggravé.

L'Égypte est le pays où la prévalence de l'hépatite $\mathrm{C}$ est la plus élevée au monde: elle serait de $13 \%$ selon l'OMS. La cause initiale de sa propagation résiderait dans la campagne de traitement de masse contre la bilharziose, menée entre 1960 et 1980. Il est désormais établi que les seringues utilisées durant cette campagne étaient mal ou pas stérilisées. Cela expliquerait que le taux de prévalence de l'hépatite $\mathrm{C}$ soit très élevé chez les adultes âgés de plus de 40 ans dans certaines régions rurales. Selon l'Inserm, ce taux peut atteindre, selon les zones, jusqu'à $50 \%$ de la population ${ }^{31}$.

Avant la mise en évidence de l'agent viral de l'hépatite $\mathrm{C}$, la presse égyptienne évoquait déjà l'existence de ce virus en Égypte, en tant qu' «hépatite non A non B». En 1989-1990, la presse a tenu un discours alarmant sur ce nouveau virus qui allait, disait-elle, "détruire le foie des Égyptiens», déjà attaqué par la bilharziose et par l'hépatite B. Ce discours était souvent accompagné de déclarations de médecins ou d'entretiens avec des spécialistes de la question comme le docteur Yasin 'Abd alGhaffar et le docteur 'Abd al-Rahman al-Zâbadi. Cependant, les instances publiques de la santé ne réagissaient pas. Il fallut attendre le milieu des années 1990 pour que l'importance de l'épidémie soit reconnue. En 1994, 'Abd al-Ghaffar fit une conférence en Arabie Saoudite où il exposa la gravité de la situation en Égypte. À

30 - Source : communiqué de presse de l'OMS du 14 mars 2002.

31 - Source : «Particularité de l'hépatite C en Égypte», Actualité de l'Inserm, n²03, novembre 2006. 
la suite de celle-ci, certains pays du Golfe exigèrent un certificat médical attestant que les Égyptiens candidats à l'immigration n'étaient pas atteints par l'hépatite C. Les autorités furent alors mises dans l'obligation d'agir, tant à cause du scandale provoqué par la déclaration du médecin qu'en raison des exigences des pays destinataires de l'immigration égyptienne ${ }^{32}$. La reconnaissance officielle de l'existence d'une maladie ou d'une épidémie est un moment crucial de sa carrière, dans la mesure où celle-ci ne devient un problème de santé publique que lorsqu'elle est devenue un problème pour les instances politiques ${ }^{33}$. Quoique dans de moindres proportions, la maladie a continué à se propager jusqu'à maintenant, notamment à cause des déficiences du système de santé dans les domaines de la transfusion sanguine et de l'hygiène médicale.

Hormis les périodes de guerre, l'Égypte n'a jamais disposé de suffisamment de sang pour traiter les malades. Elle importait donc une partie du sang destiné à la transfusion. Cependant, à la suite de la découverte du virus du sida, cette importation a été arrêtée par crainte de la contamination. Au total, les banques de sang, privées ou publiques, ne répondaient pas à plus de $25 \%$ des besoins, le reste étant comblé par des "gangs du commerce de sang", comme les nommait la presse égyptienne. La police a arrêté à plusieurs reprises des bandes organisées qui achetaient du sang aux chômeurs et aux toxicomanes, sans se préoccuper de savoir si le sang était ou non contaminé. À cette même époque, ceux qui ne pouvaient pas acheter de sang pour leurs proches malades donnaient leur propre sang, sans effectuer non plus d'analyses. Il s'ensuivait bien souvent une contamination des malades. Ainsi ce père se désolant d'avoir contaminé son fils.

\begin{abstract}
«Mon fils a subi une opération chirurgicale. Il avait besoin de sang, mais il n'y en avait pas de disponible. On m'a demandé si un membre de la famille pouvait donner du sang, c'était urgent, j'ai dit: "moi je donnerai du sang, je suis son père, je suis la personne la plus proche, c'est normal que ce soit moi". Quelque temps plus tard, j'ai appris que mon fils avait cette maudite maladie. J'ai cherché. J'ai voulu savoir comment mon fils avait eu ça. C'est un enfant que j'ai toujours protégé. Je n'ai pas trouvé la cause. Un jour, j'ai raconté au médecin que j'avais donné du sang à mon fils et que moi je ne suis pas malade. Il m'a demandé de faire des analyses de sang et c'est là que j'ai découvert que j'avais ce virus et que c'est moi qui l'avais donné à mon propre fils. J'ai voulu sauver mon fils et je lui ai donné une maladie, une maladie très grave. Depuis, [... j'essaye tout pour le sauver ${ }^{34}$.»
\end{abstract}

Certes, le ministère de la Santé s'est depuis efforcé de rendre sûre la transfusion sanguine. Toutefois, la transmission nosocomiale de l'hépatite $\mathrm{C}$ demeure toujours un problème d'actualité, à cause des carences de l'hygiène médicale. C'est ainsi qu'un jeune médecin déclare :

32 - Pour un compte rendu circonstancié, voir S. Radi, art. cit., 2006.

33 - Voir N. Dodier, op. cit., 2003.

34 - Entretien avec Ahmad F. (septembre 2005). Les conversations citées dans cet article sont issues d'un corpus, constitué durant l'été et l'automne 2005, d'une trentaine d'entretiens non directifs. Les personnes enquêtées ne constituent pas un échantillon statistiquement représentatif, mais appartiennent soit à la classe moyenne (qui est assez pauvre en Égypte), soit à la classe populaire urbaine. C'est pour cette population, de loin la plus nombreuse, que l'accès aux soins est le plus problématique. 
«Dans les milieux hospitaliers, la contamination continue. On ne fait pas attention. Lors des accouchements, on ne met pas de gants et on utilise le même matériel pour plusieurs femmes. Plusieurs médecins et infirmiers ont attrapé cette maudite maladie de cette manière. Ni le personnel médical, ni les patients ne sont à l'abri de ce fléau ${ }^{35}$.»

La grippe aviaire ne présente pas une histoire aussi longue: la maladie était connue d'emblée et inquiétait les autorités sanitaires mondiales autant que les autorités égyptiennes. Cette inquiétude était forte car l'élevage du poulet et des pigeons, qu'il soit industriel ou familial, est très développé en Égypte. La réaction des autorités sanitaires face à la première manifestation de la maladie, au début des années 2000, fut donc très rapide et prit la forme d'une éradication massive des élevages. Cette politique s'avéra pourtant assez problématique, tout d'abord parce que les élevages familiaux sont parfois l'une des seules richesses des familles pauvres et, outre la vente, leur seul moyen de manger occasionnellement de la viande. C'est ainsi que les petits éleveurs cachaient leurs poulets sous leur lit, ce qui ne faisait qu'augmenter les risques de transmission. Elle fut problématique aussi parce que la mobilisation continue de l'appareil sécuritaire et sanitaire s'avéra difficile, de sorte que les contrôles diminuèrent.

\section{Les réponses du système santé égyptien}

Les réponses du système de santé égyptien ont donc été insatisfaisantes, trop lentes après l'apparition de l'hépatite $\mathrm{C}$ et sans doute trop brutales face à la grippe aviaire. À la lenteur des réponses s'ajoute la difficile mobilisation des moyens. L'hépatite $\mathrm{C}$ en offre un exemple frappant.

Après la reconnaissance officielle de l'existence de l'épidémie, en 1994, le ministre de la Santé a en effet encouragé les chercheurs égyptiens à mettre au point un médicament national à base de plantes, dont le prix serait à la portée de la population et à la portée de l'État, puisque celui-ci n'a pas les moyens de soigner tous les malades en recourant à l'Interféron (le coût du traitement par l'Interféron peut atteindre jusqu'à 70000 livres pour un patient, ce qui occasionnerait à l'État, s'il était distribué à tous les malades, une dépense de 70 milliards de livres par an ${ }^{36}$ ). Le ministre de la Santé aurait alors passé un accord avec l'une des plus importantes institutions de recherches du pays, le Centre national de la recherche ${ }^{37}$. La position du ministère a été facilitée par la polémique ouverte en Égypte à propos de l'Interféron, considéré comme un remède faiblement efficace pour le génotype 4 , qui représente $90 \%$ des hépatites $C$ en Égypte. Cette polémique a contribué à accréditer la position des autorités égyptiennes, puisque le remède apparaissait à

35 - Entretien avec Kamal R. (septembre 2005).

36 - La livre égyptienne vaut 0,12 euro, le salaire moyen mensuel se situant autour de 400 livres, soit un peu moins de 51 euros.

37 - Le Centre national de la recherche est le principal organisme égyptien de recherche fondamentale et appliquée. Il ne s'étend toutefois pas aux sciences sociales et humaines. Les Égyptiens le désignent couramment par son acronyme anglais NRC (National Research Center). Sur le NRC et sur les institutions de recherche, voir Radi, «L'état de la science en Égypte», Études et documents du CEDEJ, $\mathrm{n}^{\circ} 18,2005$. 
la fois trop cher et d'un effet limité, voire dangereux ${ }^{38}$. La recherche locale d'un palliatif s'en est trouvée confortée. Comme les personnes atteintes par le virus de l'hépatite $\mathrm{C}$ ne disposent pas d'un remède efficace et, à plus forte raison, comme il n'existe pas en Égypte de protocole de soins bien établi, les patients, influencés par cette polémique, se soignent à l'aide d'autres palliatifs prescrits par leur médecin, comme les anti-oxydants, qui, s'ils ne sont pas censés guérir la maladie, passent pour protéger le foie de la cirrhose et du cancer, ou encore le $\mathrm{BDD}^{39}$, dite "pilule jaune», provenant de Chine. Le BDD est supposé diminuer l'augmentation des enzymes hépatiques transaminases et permettre au foie d'améliorer ses capacités de renouvellement cellulaire.

Ainsi le coût du seul médicament connu, sa réputation de faible efficacité, la particularité du VHC 4, et la nécessité de proposer malgré tout des recours aux personnes atteintes, ont abouti à stimuler une production locale, pour une part soutenue par les instances politiques, pour une part issue d'initiatives privées. Initiatives plus ou moins sérieuses, mais difficiles à mettre radicalement en cause, dans la mesure où elles découlent du retrait de l'État et de son choix assumé de promouvoir des solutions locales. Cette tendance s'est aggravée par la mise en place de simples soins, qui ne sont même plus palliatifs, mais de substitution. La "découverte» par un médecin d'une cure fondée sur les pigeons et la "découverte» d'un vétérinaire qui soigne avec le lait et l'urine de la chamelle en sont autant d'exemples.

L'un des problèmes de l'accès aux soins réside ainsi visiblement dans le financement individuel de la cure. L'Égypte possède des cliniques (privées) qui vont du simplement correct (voire moins) au luxueux, des dispensaires, des hôpitaux universitaires, des hôpitaux relevant de la sécurité sociale, des hôpitaux pour les militaires, d'autres pour la police, etc. L'offre est donc diversifiée, mais l'accès est lié à la place que l'on occupe dans la société, à l'existence d'accords professionnels, à des privilèges de fonction, et aux relations dont on dispose ou non. L'Égypte a mis en place un système de sécurité sociale auquel $42 \%$ de la population adhère. L'adhésion à ce système est obligatoire pour tous les fonctionnaires, les employés du secteur public et du secteur privé. Bénéficient aussi du système les étudiants, les enfants de la naissance à l'âge de 6 ans, les retraités et les veuves d'anciens assurés. Ceux qui ne bénéficient pas de la sécurité sociale peuvent aussi se soigner aux frais de l'État, s'ils n'ont pas les moyens de le faire à leurs frais dans le secteur privé.

Lorsque l'on entend des responsables du système de protection sociale égyptien en décrire le fonctionnement, on a l'impression qu'il est efficace. Mais, dans la

38 - Remarquons toutefois une nette évolution dans l'estimation de l'efficacité de l'Interféron. Si, durant une conférence organisée par le Centre culturel français du Caire il y a quelques années, le professeur Fontanet avait déjà estimé que 50 \% des patients égyptiens répondaient positivement au traitement, plus récemment, d'après une étude du même professeur Fontanet et du professeur Mustafa K. Muhammad, 80 \% des personnes atteintes du VHC, dans la cohorte sur laquelle ils travaillent, dans un village du Delta du Nil, auraient répondu positivement au traitement PEG-IFNa ("Particularités de l'hépatite C en Égypte», Actualité de l'Inserm, n²03, novembre 2006, www.insermactualités.com).

39 - BDD pour Biphenyl Dimethyl Dicarboxylate. 
pratique, on se rend vite compte qu'un "bénéficiaire» de la sécurité sociale ne dispose pas pour autant ipso facto de la capacité de se soigner. Toute demande de soin apparaît complexe et toute prestation qu'on sollicite devient une exception, un "service», de sorte que l'on n'est bien soigné dans les hôpitaux de la sécurité sociale que si l'on y connaît quelqu'un. Les assurés sociaux ne peuvent pas choisir le médecin ou l'hôpital pour se soigner. Souvent, les malades expriment leur défiance à l'égard des médecins qui relèvent des structures de la sécurité sociale, dépeints comme sujets à des erreurs de diagnostic et traitant mal les malades. Un jeune employé, affirme :

"I'ai la sécurité sociale, mais j'espère ne jamais avoir besoin d'y aller. Les médecins et les infirmiers traitent les malades comme des animaux. On dirait qu'on vient mendier; c'est insupportable. Il faut attendre des heures et, en plus, ils peuvent te donner n'importe quel médicament ${ }^{40}$.»

Nada, une femme d'une cinquantaine d'années, qui travaille dans une école étrangère et qui est diabétique raconte :

«On ne peut pas vraiment compter sur la sécurité sociale. J'ai beaucoup, beaucoup souffert à cause de ce système. Normalement, on devait me donner l'insuline, sinon je ne peux pas vivre. Je suis allée plusieurs fois la demander. On m’a réclamé des certificats médicaux et plein d'autres papiers, mais chaque fois on me disait qu'il n'y avait pas de médicaments, ou que le médecin n'était pas encore là, ou qu'il fallait revenir un autre jour. Une fois, une amie m'a dit: "si tu veux avoir tes médicaments, il faut d'abord aller voir le médecin de la sécurité sociale dans son cabinet". J'y suis allée et j'ai payé 80 livres la consultation, et après j'ai eu le médicament. Parfois on me dit qu'il n'y a plus d'insuline et on me donne des cachets à la place. Quand je peux acheter mon médicament, je l'achète, et quand je ne peux pas, je prends les cachets en attendant ${ }^{41}$.»

Le cas de la grippe aviaire est différent, puisqu'il ne s'agit pas d'une épidémie mais de la crainte d'une mutation du virus liée à la concentration d'élevages de poulets dans un milieu humain vivant en symbiose avec cet élevage. On ne dénombre actuellement qu'un peu plus d'un vingtaine de cas. De ce point de vue, le système de santé n'est pas concerné en profondeur. Il s'agit de prévenir un risque mais non de gérer une crise. De plus, la mobilisation internationale paraît plus forte, dans la mesure où le risque est direct pour les autres pays et où les moyens à consacrer en Égypte à cette maladie sont plus faibles qu'en ce qui concerne l’hépatite C.

\section{Santé et société}

On conclura en soulignant la relation étroite entre l'état du système de santé et la forme de l'épidémie. La dynamique de l'hépatite $\mathrm{C}$ ne s'explique pas par la nature du virus mais par les conditions sociales de sa transmission. C'est l'état du système de santé qui, en quelque sorte, fait la niche écologique de l'épidémie. Les mauvaises conditions d'hygiène prévalant dans les structures sanitaires expliquent la large diffusion de l'atteinte, et la faiblesse du système de sécurité sociale entraîne la mise en place de soins paillatifs sans grande efficacité. En tout état de cause, ces 
soins ne sont pas l'expression d'une culture différente, rétive aux techniques de la biomédecine; ils sont la simple expression d'un manque. Il en est de même de la grippe aviaire: les limites des précautions sanitaires brutalement prises par les autorités égyptiennes résident dans l'absence d'une indemnisation financière des éleveurs. Le coût social d'une telle politique est excessif.

Étudier les systèmes de santé des pays du Sud implique donc beaucoup moins l'étude de leurs particularités culturelles - c'est-à-dire la réduction à l'anthropologique - que l'étude des limites de leurs ressources et de leur capacité à faire face au risque sanitaire. Sans réduire non plus la maladie à sa dimension sociale, force est de constater que la carrière d'une maladie, notamment épidémique, dépend pour beaucoup de l'état de la société et de son système de santé. Ce dernier n'est pas toujours à même de défendre la population contre l'épidémie : il peut, paradoxalement, être la niche écologique où se développe la maladie.

\section{Bibliographie}

- Augé M, «L'anthropologie de la maladie», L'Homme, vol. 26, $\mathrm{n}^{\circ} 1-2,1986$.

- Bado J.-P., Médecine coloniale et grandes endémies en Afrique, Paris, Karthala, 1996.

- Bеск U., La société du risque. Sur la voie d'une autre modernité, Paris, Aubier, 2001.

- Bungener M., "Canicule estivale : la triple vulnérabilité des personnes âgées", Mouvements, $\mathrm{n}^{\circ} 32,2004$.

- Buton F., «Sida et politique: saisir les formes de la lutte», Revue française de science politique, vol. 55, n5-6, 2005.

- Cassuto J.-P., De la maladie de la vache folle à celle de Creutzfeldt-Jakob, Paris, Odile Jacob, 1999.

- Chastel C., Ces virus qui détruisent les hommes, Paris, Ramsey, 1996.

- Delaporte F., Histoire de la fièvre jaune. Naissance de la médecine tropicale, Paris, Payot, 1989.

- Dodier, N., Les leçons politiques de l'épidémie de sida, Paris, Éditions de l'EHESS, 2003.

- Epstein S., Histoire du sida, Paris, Les Empêcheurs de penser en rond, 2001.

- Fainzang S., Pour une anthropologie de la maladie en France : un regard africaniste, Paris, Éditions de l'EHESS, 1989.
- Fassin D., L'espace politique de la santé. Essai de généalogie, Paris, PUF, 1996.

- Godard O., «Le principe de précaution comme norme de l'action publique, ou la proportionnalité en question", Revue économique, vol. 54, $\mathrm{n}^{\circ}$ 6, 2003.

- Grmek M., Histoire du sida, Paris, Payot, 1989.

- Gualde N., Épidémies, la nouvelle carte, Paris, Desclée de Brouwer, 2002.

- Hamdouch A. et M.-H. Depret, «Carences institutionnelles et rationnement de l'accès à la santé dans les pays en développement : repères et enjeux", Mondes en Développement, vol. 33, $\mathrm{n}^{\circ} 131,2005$.

- Hermet G., «Un régime à pluralisme limité. À propos de la gouvernance démocratique», Revue française de science politique, vol. 54, $\mathrm{n}^{\circ} 1,2004$.

- Herzlich C, «Vingt ans après... l'évolution d'une épidémie», Études, t. 396, n² 2, 2002.

- Hours B., "Pour une anthropologie de la santé en sociétés ", in B. Hours (dir.), Systèmes et politiques de santé. De la santé publique à l'anthropologie, Paris, Karthala, 2001.

- Massé R., «La santé publique comme projet politique et projet individuel», in B. Hours (dir.), Systèmes et politiques de santé. De la santé publique à l'anthropologie, Paris, Karthala, 2001. 
- Milet M., «Cadre de perception et luttes d'imputation dans la gestion de crise: l'exemple de la canicule d'août 2003 ", Revue française de science politique, vol.55, $\mathrm{n}^{\circ} 4$, 2005.

- Pinell P., Une épidémie politique. La lutte contre le sida en France (1981-1996), Paris, PUF, 2002.

- Radi S., "L'état de la science en Égypte», Études et documents du CEDEJ, $\mathrm{n}^{\circ}$ 18, 2005.

- Radi S., «Débats de presse, scandale et mise en place d'une politique de prévention : à propos de l'hépatite $\mathrm{C}$ en Égypte», Revue d'épidémiologie et de santé publique, vol. 54, 2006.

- RADI S, «L'hépatite C et les défaillances du système égyptien de santé publique. Itinéraires thérapeutiques et solutions palliatives», Égypte-Monde arabe, n 4, 2007.
- Seguin E., "La crise de la vache folle au Royaume-Uni. Quelques explications", Revue française de science politique, vol.52, $\mathrm{n}^{\circ} 2-3,2002$.

- Seguin E., «L'évaluation britannique du risque de transmission de la maladie de la vache folle aux humains", Revue française d'administration publique, $\mathrm{n}^{\circ} 103$, 2002/2003.

- Tizio S., «État de santé et système de soins dans les pays en développement : la contribution des politiques de santé au développement durable», Mondes en développement, vol. $32, \mathrm{n}^{\circ} 127,2004$.

- Tizio S., «Trajectoires socioéconomiques de la régulation des systèmes de santé dans les pays en développement : une problématique institutionnelle», Mondes en développement, vol.33, $\mathrm{n}^{\circ}$ 131, 2005. 